\title{
Galectin-7 is elevated in endometrioid (type I) endometrial cancer and promotes cell migration
}

\author{
ELLEN MENKHORST ${ }^{1,2}$, MEAGHAN GRIFFITHS ${ }^{1,2}$, MICHELLE VAN SINDEREN $^{1,2}$, \\ KATE RAINCZUK $^{1,2}$, KEITH NIVEN $^{3}$ and EVDOKIA DIMITRIADIS ${ }^{1,2}$ \\ ${ }^{1}$ Centre for Reproductive Health, Hudson Institute of Medical Research, Clayton, VIC 3168; \\ ${ }^{2}$ Department of Molecular and Translational Medicine, Monash University; \\ ${ }^{3}$ FlowCore, Technology Research Platforms, Monash University, Clayton, VIC, 3800, Australia
}

Received March 14, 2018; Accepted June 7, 2018

DOI: $10.3892 / \mathrm{ol} .2018 .9193$

\begin{abstract}
Endometrial cancer (EC) is the most commonly diagnosed gynecological malignancy in Australian women. Notably, its incidence and mortality rate is increasing. Despite this, there are limited treatment options for EC. Galectin-7 regulates tumorigenesis in numerous epithelial cancer types, but the role of galectin-7 has not been investigated in EC. It was hypothesized that galectin-7 expression would be altered in EC and contribute to the development of EC. Galectin-7 levels in EC and benign endometrium were quantified by reverse transcription-quantitative polymerase chain reaction (RT-qPCR) and ELISA. The effect of recombinant galectin-7 $(1 \mu \mathrm{g} / \mathrm{ml})$ on cell adhesion, proliferation, apoptosis (xCELLigence and flow cytometry), migration (wound healing assay) and gene expression (RT-qPCR) was investigated using three human EC cell lines (Ishikawa, HEC1A and AN3CA). Galectin-7 gene and protein expression was significantly elevated in Grade 3 EC, compared with benign tissues. Galectin-7 was almost undetectable in Ishikawa and AN3CA cells, but highly expressed by HEC1A cells. Recombinant galectin-7 had no significant effect on cell proliferation or apoptosis in any cell line, but significantly reduced cell adhesion in Ishikawa (at 4 and $6 \mathrm{~h}$ ) and AN3CA (at 2, 3, 4 and $6 \mathrm{~h})$. Galectin-7 significantly promoted Ishikawa migration and significantly elevated collagen type IV $\alpha 1$ chain and intercellular adhesion molecule 1 (ICAM1) gene expression during wound healing. The present study demonstrated that galectin-7 production increased in EC with increasing cancer grade; therefore, galectin-7 may promote the metastasis of EC by reducing cell-cell adhesion and enhancing cell migration.
\end{abstract}

Correspondence to: Professor Evdokia Dimitriadis, Centre for Reproductive Health, Hudson Institute of Medical Research, 27-31 Wright Street, Clayton, VIC 3168, Australia

E-mail: evdokia.dimitriadis@hudson.org.au

Key words: endometrial cancer, uterus, galectin-7, migration, wound-healing, intercellular adhesion molecule 1, collagen type IV $\alpha 1$ chain, Ishikawa, AN3CA, HEC1A

\section{Introduction}

Endometrial cancer (EC) is the most common gynecological cancer diagnosed in Australian women (1). Notably, the incidence and mortality rate of EC is increasing by an estimated $1-2 \%$ annually (2), which is partially due to the increasing life expectancy and obesity levels (3). Despite this, there are limited treatment options for EC, particularly for the recurrent or metastatic disease (2).

EC can be divided into two histologic types: Type 1; and Type 2. Type 1 carcinoma types account for $\sim 85 \%$ of EC cases (4) and are primarily endometrioid in histology, of a lower grade and confined to the uterus at diagnosis (2). Type 1 tumor types are characteristically estrogen-mediated and associated with $K$-ras and phosphatase and tensin homolog (PTEN) loss or mutation (2). Type 2 non-estrogen carcinoma types are primarily non-endometrioid in histology, higher-grade adenocarcinomas and contain tumor protein P53 (p53) mutations (2); however, there is significant heterogeneity and overlap between these two types of EC and the characteristics of each type are not always limited to the one type of EC (2).

Type $1 \mathrm{EC}$ is frequently preceded by endometrial hyperplasia, whereby the endometrial glands undergo excess proliferation, resulting in an increase in the glandular:stroma ratio (4). Hyperplasia is primarily associated with unopposed estrogen stimulation, or it may be due to specific genetic alterations (4). Staging of type $1 \mathrm{EC}$ is according to guidelines from the International Federation of Gynecology and Obstetrics (5), with tumor grade (Grade 1-3) based on histological similarity to normal endometrium, metastatic behavior and the extent of invasion into the uterine corpus and surrounding peritoneum (4).

Galectins are animal lectins that bind to surface glycoproteins, in particular $\beta$-galactoside (6). Galectins regulate numerous cell functions critical for cancer progression, including elevated cell proliferation, cell adhesion and migration, apoptosis and immune suppression (7). Furthermore, the cell-cell and cell-matrix interactions exhibited by galectins and their high affinity for specific oligosaccharides make galectins promising markers and/or therapeutic targets for cancer (8). The expression and function of galectins in EC prognosis and progression have not been well investigated to date, although 
galectin-3 production in epithelial cancer cells is significantly associated with poor prognosis and reduced survival rates 5 years after diagnosis (9).

Galectin-7 is a $15 \mathrm{kDa}$ prototype galectin expressed specifically by stratified epithelial cells (8). Similar to all galectins, galectin-7 is secreted (10) without a typical secretion signal peptide sequence, and it is released from cells via a route that requires intact carbohydrate-binding activity (11). Galectin-7 generally localizes to areas of cell-cell contact (6), and has a well-characterized role in wound healing. Additionally, it accelerates the re-epithelialization of corneal wounds more efficiently than the majority of known growth factors $(12,13)$. In the uterus, galectin-7 promotes wound repair following menstruation (14) and elevated endometrial epithelial galectin-7 is associated with miscarriage, possibly due to its role as an anti-adhesion molecule during implantation (15).

The role of galectin-7 in cancer may be tissue specific. Altered galectin-7 expression has been determined in a number of cancer types, including cervical, breast and ovarian cancer $(7,8)$. The role of galectin-7 in cancer can be pro- or anti-tumor: In breast cancer, galectin-7 is pro-tumor, enhancing spontaneous metastatic capability and protecting cancer cells from apoptosis (16). Galectin-7 is a negative prognostic marker for ovarian cancer (17) and renal clear-cell carcinoma (18). Conversely, galectin-7 may exhibit anti-tumor properties in other types of cancer; for example, it is absent in prostate cancer cells (19), reduced in gastric cancer (20) and causes prostate and DLD-1 colon cancer cells to have greater sensitivity to apoptosis $(19,21)$.

The role of galectin-7 in EC has not been investigated. We hypothesized that galectin-7 expression would be altered in human EC, similar to other epithelial malignancy types, and contribute to the development of EC. The aim of the present study was to determine the expression of galectin-7 in type 1 human endometrioid EC across Grades 1-3 and normal endometrium. The effect of elevated galectin-7 on Ishikawa, HEC1A and AN3CA (endometrial epithelial cancer cell lines) cell proliferation, apoptosis, migration and gene expression was determined.

\section{Materials and methods}

Patient samples. The present study was approved by the Monash Health Human Research and Ethics Committee (approval no. 06014C) and the Victorian Cancer Biobank (Project no. 13018). Written and informed consent was obtained from each patient.

The Victorian Cancer Biobank (Melbourne, Victoria, Australia) provided RNA (for qPCR) from EC ( $\mathrm{n}=7-8 /$ grade) or benign post-menopausal endometrium $(n=7)$ whole tissue, and endometrial biopsies (for protein extraction) from EC $(n=9 /$ grade $)$ or benign endometrium $(n=9)$. The mean and median age for all patients was: mean $58.4 \pm 1.9$ years; median 59 years. Patient age distribution is provided in Table I. Samples were collected in Melbourne, Victoria, Australia between 2007 and 2014.

Proliferative phase pre-menopausal endometrium $(n=4$; Table I) was collected by curettage from females between day 7 and 13 of their menstrual cycle that were scheduled for tubal ligation, as a non-tumor control group. Biopsies were examined by a blinded experienced gynaecological pathologist (Melbourne Pathology, Collingwood, Victoria, Australia; Anatpath, Gardenvale, Victoria, Australia) to confirm that they exhibited no evidence of possible endometrial dysfunction. Females had no steroid treatment or other medication for at least 2 months prior to tissue collection.

Cell culture. All cell cultures were conducted in a humidified incubator maintained at $37^{\circ} \mathrm{C}$ in an atmosphere containing $5 \% \mathrm{CO}_{2}$. Grade (G)-1 EC derived Ishikawa cells were provided by Dr M. Nishida (Tsukuba University, Tsukuba, Japan) in 2014 and cultured in phenol red-free Dulbecco's modified Eagle's medium (DMEM; Gibco, Thermo Fisher Scientific, Inc., Waltham, MA, USA) with $10 \%$ fetal calf serum (FCS; Gibco, Thermo Fisher Scientific, Inc.). G2 derived HEC1A [authenticated by Monash Health Translation Precinct (MHTP) Medical Genomics Facility (Clayton, Victoria, Australia) in 2016] were purchased from the American Type Culture Collection (ATCC; Manassas, VA, USA) in 2012 and cultured in McCoy's medium (Gibco, Thermo Fisher Scientific, Inc.) with 10\% FCS. G3 derived AN3CA (authenticated by MHTP Medical Genomics Facility in 2012) were purchased from ATCC in 2012 and cultured in DMEM medium (containing phenol red; Gibco, Thermo Fisher Scientific, Inc.) with 10\% FCS.

$R N A$ preparation and reverse transcription-quantitative polymerase chain reaction ( $R T-q P C R)$. Total RNA was isolated from cancer cell lines using TriReagent (Sigma-Aldrich; Merck KGaA, Damstadt, Germany). Contaminating genomic DNA was digested using the DNAfree kit (Ambion; Thermo Fisher Scientific, Inc.), according to the manufacturer's protocols. To test the RNA yield, purity and concentration, $2 \mu \mathrm{l}$ was analyzed using a NanoDrop spectrophotometer (Thermo Fisher Scientific, Inc.) at an absorbance ratio of A260/280 nm. cDNA was synthesized from total RNA (250 ng) using Superscript III First-Strand Synthesis system (Invitrogen; Thermo Fisher Scientific, Inc.).

RT-qPCR analyses of cancer cell lines and cancerous, benign and proliferative endometrial tissue were performed on the ABI 7500HT fast block Real-Time qPCR system (Applied Biosystems; Thermo Fisher Scientific, Inc.) in triplicate (final reaction volume, $10 \mu \mathrm{l}$ ) in 384-well Micro-Optical plates (Applied Biosystems; Thermo Fisher Scientific, Inc.). For each sample, $25 \mathrm{ng}$ cDNA was added to a PCR mix made with the 2X Fast-Start SYBR-Green master mix containing ROX passive reference dye (Applied Biosystems; Thermo Fisher Scientific, Inc.) and $10 \mathrm{nM}$ primers. The primer sequence details are listed in Table II. A template-free negative control in the presence of primers and RNase-free water, and RNase-free water only (for negative controls) were added for each run. The qPCR protocol was as follows: $95^{\circ} \mathrm{C}$ for $10 \mathrm{~min}$; 40 cycles of $95^{\circ} \mathrm{C}$ for $15 \mathrm{sec}$; followed by $60^{\circ} \mathrm{C}$ for $1 \mathrm{~min}$. Relative expression levels were calculated by the comparative cycle quantification method $(\Delta \Delta \mathrm{Cq})(22)$ as outlined in the manufacturer's protocols, with 18s ribosomal RNA serving as the endogenous control for normalization.

Protein extraction. Endometrial tissue (benign and cancer) was mechanically homogenized using the QIAGEN TissueLyser LT machine (Qiagen, Chadstone, Victoria, Australia) in ice-cold 
Table I. Age of patients with endometrial cancer.

\begin{tabular}{lccccc}
\hline Age $(\mathrm{n})$ & Proliferative & Benign & Grade 1 & Grade 2 & Grade 3 \\
\hline RNA & - & N/A $(7)$ & $50.9+4.2(7)^{\mathrm{a}}$ & $63.8+5.1(8)^{\mathrm{a}, \mathrm{b}}$ & $70.1+4.4(7)^{\mathrm{b}}$ \\
Protein & $41.7+1.9(4)^{\mathrm{p}}$ & $41.3+1.9(9)^{\mathrm{p}}$ & $57.6+4.3(9)^{\mathrm{q}}$ & $60.6+3.4(9)^{\mathrm{q}}$ & $73.5+2.3(9)^{\mathrm{r}}$ \\
\hline
\end{tabular}

Data shows age in years and sample size (n). A significant difference in age is indicated by the superscript letter: A different letter indicates the groups are statistically different. Ages were not compared between the RNA and protein sample sets. N/A, not available.

Table II. Primer sequences.

\begin{tabular}{|c|c|c|}
\hline Primer & Forward sequence $\left(5^{\prime}-3^{\prime}\right)$ & Reverse sequence (5'-3') \\
\hline Col4 $\alpha 1$ & AAAGGGAGATCAAGGGATAG & TCACCTTTTTCTCCAGGTAG \\
\hline ICAM1 & GCCGGCCAGCTTATACACAA & TGGCCACGTCCAGTTTCC \\
\hline TGF $\beta 1$ & AACCCACAACGAAATCTATG & СTTTTAACTTGAGCCTCAGC \\
\hline ITG $\alpha 2$ & TTAGGTTACTCTGTGGCTGCAAT & AGGAGCACCAGCAACAAAGTG \\
\hline $18 \mathrm{~s}$ & GATCCATTGGAGGGCAAGTCT & CCAAGATCCAACTACGAGCTT \\
\hline
\end{tabular}

Col4 $\alpha 1$, collagen type IV $\alpha 1$ chain; ICAM1, intercellular adhesion molecule 1 ; TGF $\beta 1$, transforming growth factor $\beta 1$; ITG $\alpha 2$, integrin subunit $\alpha 2$.

universal lysis buffer [50 mM Trizma Base (Sigma-Aldrich; Merck KGaA) pH 7.4, $150 \mathrm{mM} \mathrm{NaCl}, 2$ mM EDTA, $2 \mathrm{mM}$ EGTA, 25 mM NaF, 0.2\% Triton X-100 (Sigma-Aldrich; Merck KGaA) and 0.3\% Nonidet P-40 (Sigma-Aldrich; Merck $\mathrm{KGaA})]$ containing Protease Inhibitor Mixture Set III (1:500; EMD Millipore, Billerica, MA, USA) and stored at $-80^{\circ} \mathrm{C}$. The protein concentration was assayed by a commercial kit (Pierce BCA Protein assay kit; Thermo Fisher Scientific, Inc.).

Galectin-7 ELISA. The concentration of galectin-7 in EC biopsies and EC cell line (Ishikawa, HEC1A and AN3CA) cell lysate and conditioned media was assayed using a Human Galectin-7 ELISA kit (cat. no. ELH-Galectin7-001; RayBiotech, Inc., Norcross, GA, USA), according to the manufacturer's protocols $(15,23)$. For the cell lines, cells were cultured in serum-free media for $48 \mathrm{~h}$ prior to cell lysate being collected for the ELISA. Briefly, $30 \mu \mathrm{g}$ (Grade 3 EC only) or $50 \mu \mathrm{g}$ protein (all other lysates) was incubated at room temperature on a rotating shaker for $2 \mathrm{~h}$ prior to binding was detected according to the manufacturer's protocols. Absorbance was measured at $450 \mathrm{~nm}\left(\right.$ ClarioStar $^{\circledR}$; BMG LabTech $\mathrm{GmbH}$, Ortenberg, Germany). The minimum detection limit of the kit according to the manufacturer was $41.5 \mathrm{pg} / \mathrm{ml}$ galectin- 7 .

$x$ CELLigence real time cell adhesion and proliferation assay. Experiments were carried out using the RTCA DP xCELLigence instrument (ACEA Biosciences Inc, In vitro Technologies, Noble Park, Victoria, Australia). Cells (Ishikawa, AN3CA and HEC1A) were seeded in E-plate 96 (ACEA Biosciences) at 10,000 cells/well in the corresponding medium supplemented with $5 \%$ FCS and the plate was monitored every $1 \mathrm{~min}$ for $5 \mathrm{~h}$, then every $15 \mathrm{~min}$ for a total of $72 \mathrm{~h}$ (24). E-plates were used a maximum of 3 times, with the wells trypsinized and washed with PBS between each use, as previously described (25). Data was calculated using RTCA software 1.2, supplied with the instrument (ACEA Biosciences, San Diego, CA, USA; https://www.aceabio.com/products/xcelligence-rtca/) and exported for statistical analysis. Recombinant galectin-7 (R\&D Systems, Inc., Minneapolis, MN, USA) was added at a concentration of $1 \mu \mathrm{g} / \mathrm{ml}$, and $0.1 \%$ bovine serum albumin (BSA; final concentration 0.0005\%; Sigma-Aldrich; Merck $\mathrm{KGaA}$ ) was included as a vehicle control.

Flow cytometry. As there was no effect of galectin-7 on HEC1A cell index (by xCELLigence assay above) only Ishikawa and AN3CA cell cycle was assessed by Flow Cytometry. Ishikawa and AN3CA cells were seeded at 50,000 cells/well in a 48 -well plate and grown to $50 \%$ confluence. Cells were treated with recombinant galectin-7 or vehicle control as described previously (1ug/ml galectin-7 or $0.1 \%$ BSA); for $24 \mathrm{~h}$ (AN3CA) or $48 \mathrm{~h}$ (Ishikawa) at $37^{\circ} \mathrm{C}$. Cells were trypsinized, pelleted and fixed in $70 \%$ ethanol at $-20^{\circ} \mathrm{C}$ degrees for up to one week prior to analysis. Cells were stained with FxCycle PI/RNase staining solution (cat. no. F10797; Molecular Probes; Thermo Fisher Scientific, Inc.) and analyzed with a BDFACSCanto II flow cytometer (BD Biosciences, North Ryde, New South Wales, Australia). Cell cycle and apoptosis analyses, and model fitting, were performed with FlowJo (Version X; FlowJo LLC, Ashland, OR, USA).

Wound healing migration assay. Ishikawa cells were chosen from experiments above as being a representative EC cell line with low galectin-7 production and used to investigate the effect of elevated galectin-7 on cell migration. Ishikawa cells were seeded at 50,000 cells/well in a 48-well plate and grown to $100 \%$ confluence. Cells were wounded using a 
vacuum suction through a $200 \mu 1$ pipette tip. On the day of wounding, at designated time $0(0 \mathrm{~h})$, the cell wounds were imaged (x40 magnification) with a Motic AE31 inverted light microscope (Motic Asia, Kowloon, Hong Kong) and camera (Moticam 2500; Motic Asia) using Motic Images plus2.0 software (Motic Microscopy; https://www.motic. com/As_Support_Download/). Wounded cells were treated with $1 \mu \mathrm{g} / \mathrm{ml}$ galectin-7 or vehicle control $(0.1 \%$ BSA) for $48 \mathrm{~h}$ at $37^{\circ} \mathrm{C}$. To assess differences in wound repair, the area of each wound was manually outlined and quantitated using Image J (v10.2; National Institutes of Health, Bethesda, MD, USA) software, at 0,24 and $48 \mathrm{~h}$. These time points were selected due to a clear wound boundary being distinguishable, as the wound was not fully repaired. Data was expressed as the percentage repair at 24 and $48 \mathrm{~h}$, vs. the $0 \mathrm{~h}$ time point. For each experiment, wounds were performed and assessed in quadruplicate and repeated in 4 separate experiments.

To investigate gene expression during wound repair, Ishikawa cells were seeded at 10,000 cells/well in a 96-well plate and grown to $100 \%$ confluence. Cells were wounded and treated as aforementioned for $24 \mathrm{~h}$ prior to RNA being extracted as aforementioned.

Statistics. All statistical analyses were performed on raw data using GraphPad Prism 6 (GraphPad Software, Inc., La Jolla, CA, USA). Paired Student's t-tests, and one- and two-way analysis of variance were used as appropriate. All data is presented as mean \pm standard error of the mean and as $\%$ change from control only for graphical presentation to account for variability in wound size at time $0 . \mathrm{P}<0.05$ was considered to indicate a statistically significant difference.

\section{Results}

Galectin-7 mRNA and protein levels are elevated in Type I endometrioid cancer with increasing tumor grade. Galectin-7 mRNA (Fig. 1A) and protein (Fig. 1B) were quantitated using RT-qPCR and ELISA, respectively. Galectin-7 mRNA levels were low in all samples tested, but increased with cancer grade, and was significantly increased in Grade 3, compared with benign tissue (Fig. 1A; $F_{3,25}$ 3.181; $\mathrm{P}<0.05$ ). Galectin-7 protein was almost undetectable in proliferative phase and benign endometrium tissues (Fig. 1B), but increased with increasing cancer grade, and was significantly increased in Grade 3 tissues, compared with benign and Grade 2 tissues (Fig. 1B; $\mathrm{F}_{4,35}$ 3.401; $\mathrm{P}<0.05$ ).

In EC cell lines, galectin-7 protein was almost undetectable in AN3CA cells $(0.375 \pm 0.09 \mathrm{pg} / \mu \mathrm{g})$, expressed at low levels in Ishikawa cells $(2.365 \pm 0.39 \mathrm{pg} / \mu \mathrm{g})$ and highly expressed in HEC1A cells $(72.24 \pm 32.37 \mathrm{pg} / \mu \mathrm{g})$ (Fig. 1C).

Galectin-7 decreases Ishikawa cell adhesion and enhances cell migration. To determine the functional effect of galectin-7 on adhesion, proliferation and apoptosis, Ishikawa (Fig. 2A-C), AN3CA (Fig. 2D-F) and HEC1A (Fig. 2G and H) cells were treated with recombinant galectin-7 $(1 \mu \mathrm{g} / \mathrm{ml})$ and assayed by xCELLigence (adhesion, proliferation) or flow cytometry (proliferation, apoptosis). Galectin-7 treatment reduced the cell index (a measure of cell attachment) during the period of cell-plate adhesion (up to 6 h) of Ishikawa (4-6 h; Fig. 2A)

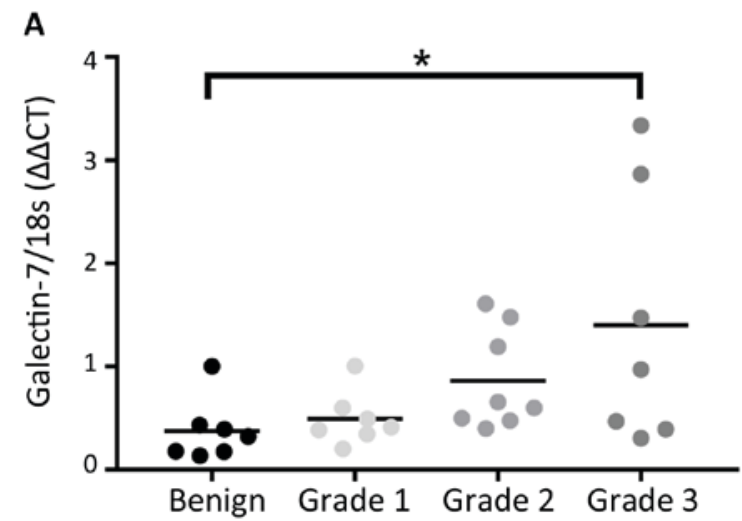

B
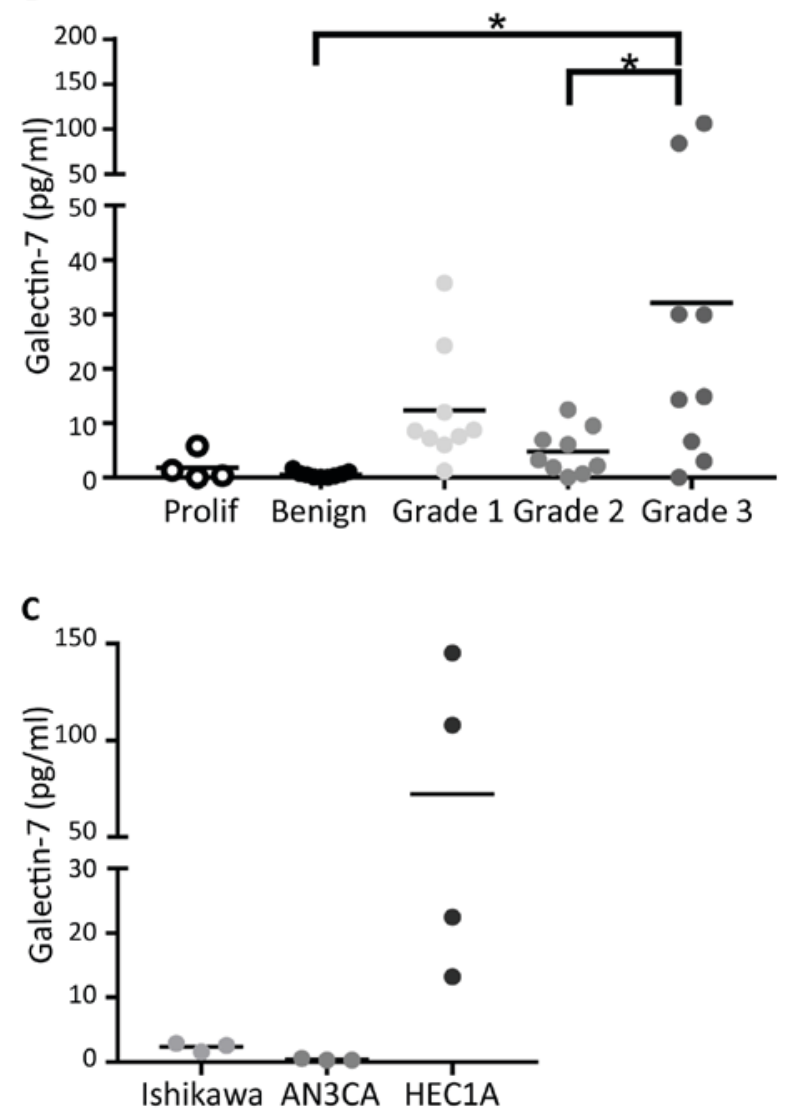

Figure 1. Galectin-7 production increases with increasing EC grade. (A) Galectin-7 mRNA production by benign post-menopausal endometrium and EC from Grades 1, 2 and 3. (B) Galectin-7 protein production by proliferative phase endometrium, benign post-menopausal endometrium and EC from Grades 1,2 and 3. (C) Galectin-7 protein production by EC cell lines after $48 \mathrm{~h}$ of culture in serum-free media. Significant difference was calculated by one-way analysis of variance; ${ }^{*} \mathrm{P}<0.05$. EC, endometrial cancer; prolif, proliferative phase.

and AN3CA cells (2-6 h; Fig. 2D) compared with the control. Cell index was also reduced during the period of proliferation (12-72 h) in Ishikawa cells (36-72 h; Fig. 2B) and AN3CA cells (18-72 h; Fig. 2E) ( $\mathrm{P}<0.05$; $\mathrm{n}=4$ /group). Conversely, galectin-7 had no significant effect on HEC1A cell index at any time-point (Fig. $2 \mathrm{G}$ and $\mathrm{H} ; \mathrm{n}=4$ /group). To determine whether the reduced cell index reflected reduced proliferation or increased apoptosis, flow cytometry was performed on Ishikawa and AN3CA cells treated with galectin-7; however, 
A

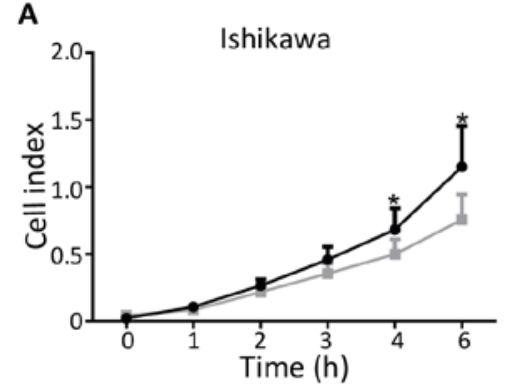

B
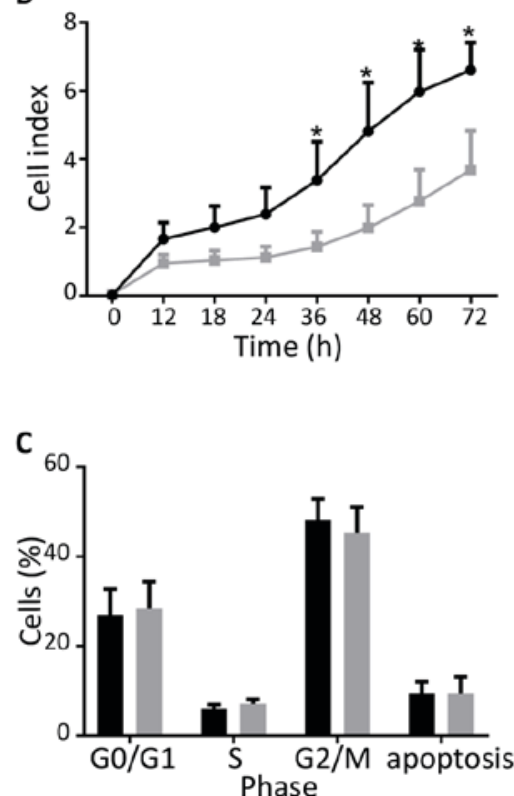

D

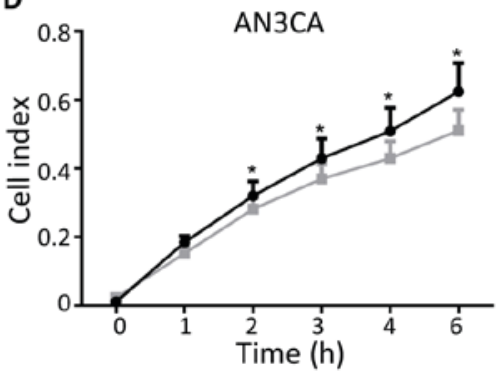

E

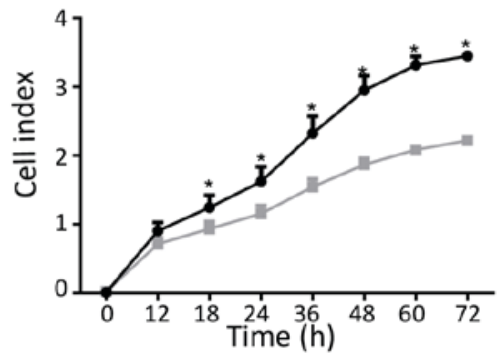

F

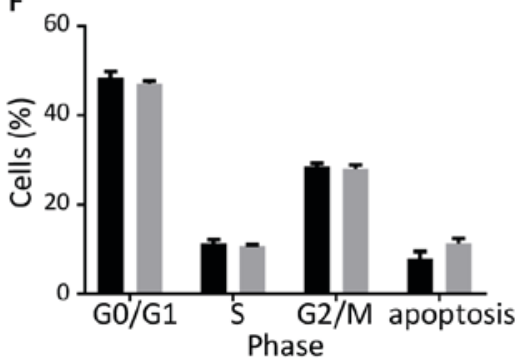

G

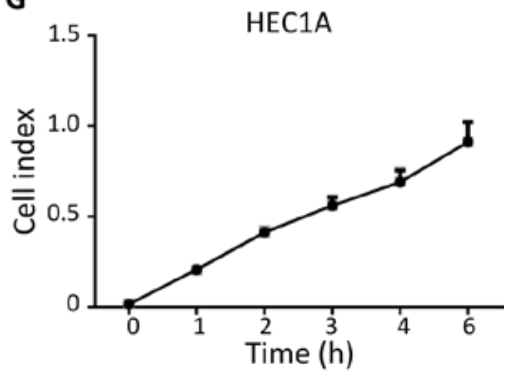

H

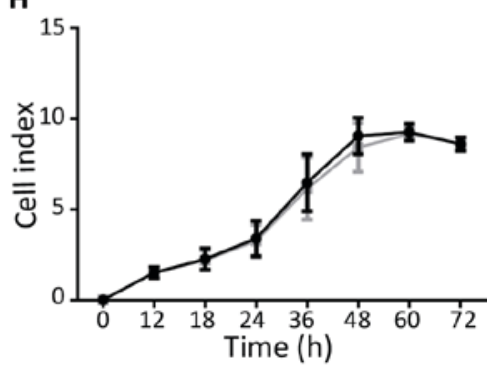

Control

Galectin-7

Figure 2. Galectin-7 reduces EC cell line adhesion but has no significant effect on proliferation or apoptosis in vitro. Ishikawa (A-C; $\mathrm{n}=4$ ), AN3CA (D-F; $\mathrm{n}=4$ ) and HEC1A (G and H; n=3) EC cell lines were treated with $1 \mu \mathrm{g} / \mathrm{ml}$ galectin-7 (grey) or vehicle control (black) and subjected to xCELLigence real-time cell analysis assays $(\mathrm{A}, \mathrm{B}, \mathrm{D}, \mathrm{E}, \mathrm{G}$ and $\mathrm{H})$ or flow cytometry $(\mathrm{C}$ and F). For the xCELLigence assays, the cell index is a measure of cell attachment. Ishikawa (A and B) and AN3CA (D and E) cell attachment was significantly reduced at almost all time-points examined; however, there was no significant effect on HEC1A cell attachment (G and $\mathrm{H})$ at any time point. Flow-cytometry demonstrated that galectin-7 had no significant effect on Ishikawa (C) or AN3CA (F) cell cycle or apoptosis. Significant difference was calculated by two-way analysis of variance, ${ }^{*} \mathrm{P}<0.05$; data are depicted as the mean \pm standard error of the mean. EC, endometrial cancer.

no significant effect of galectin-7 was determined on cell cycle or apoptosis in either cell line (Fig. 2C and F), indicating that the lower cell index seen in Ishikawa and AN3CA cells throughout the assay was due to reduced cell adhesion.

To migrate or invade, cells must first de-attach, then re-attach. To investigate whether the reduced cell adhesion observed in the xCELLigence assay would affect cell migration, wound-healing assays were performed on Ishikawa cells (Fig. 3A). Galectin-7 treatment $(1 \mu \mathrm{g} / \mathrm{ml})$ significantly enhanced Ishikawa wound closure after 24 and $48 \mathrm{~h}$ compared with the control (Fig. 3B; $\mathrm{P}<0.05 ; \mathrm{n}=4$ /group).

Galectin-7 increases collagen type IV a 1 chain (COL4A1) and intercellular adhesion molecule 1 (ICAM1) expression. To investigate the mechanism by which galectin-7 reduced cell adhesion and enhanced migration, the effect of galectin-7 treatment on the expression of a variety of factors involved with cell adhesion and migration was assessed by RT-qPCR (Fig. 4). Galectin-7 treatment $(1 \mu \mathrm{g} / \mathrm{ml})$ for $24 \mathrm{~h}$ significantly elevated Ishikawa expression of COL $4 \alpha 1$ (Fig. 4A; $\mathrm{P}<0.05$; n=4/group) and ICAM1 (Fig. 4B; P<0.05; n=4/group), although transforming growth factor $\beta 1$ (TGF $\beta 1$ ) and integrin subunit $\alpha 2$ (ITG $\alpha 2$ ) levels did not change significantly (Fig. 4C and D). No effect of galectin-7 treatment was determined on the expression of galectin-7, or the epithelial-mesenchymal transition-associated genes epithelial-cadherin, TWIST1 or TWIST2 (data not shown).

\section{Discussion}

To the best of our knowledge, this is the first study to identify and characterize a role for galectin-7 in EC. It was demonstrated that tissue galectin-7 levels increased with increasing EC grade. In vitro, the experiments indicated that increasing galectin-7 would promote EC cell tumorigenesis by reducing EC adhesion and enhancing EC cell migration. It was also determined that galectin-7 had no significant effect on proliferation or apoptosis.

Galectin-7 has tissue specific pro- or anti-tumorigenic actions. It was demonstrated that in EC, galectin-7 may be pro-tumorigenic, via its actions to promote cell migration. This has also been observed in breast (16), ovarian (26) and 
A
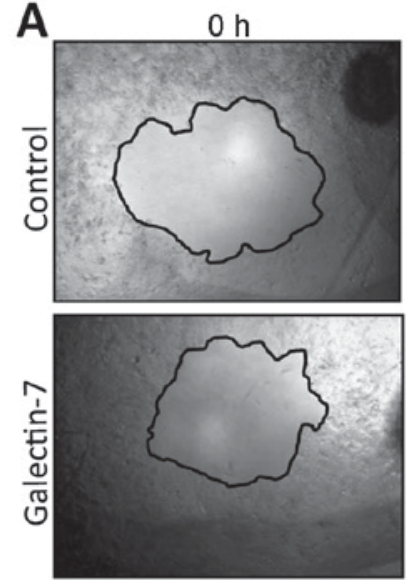

$24 \mathrm{~h}$

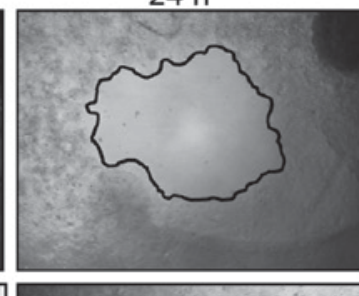

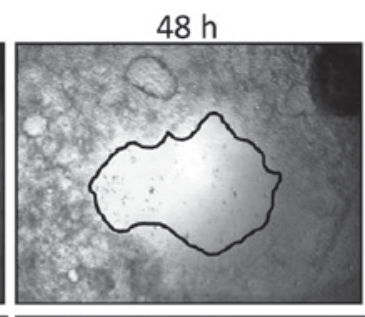
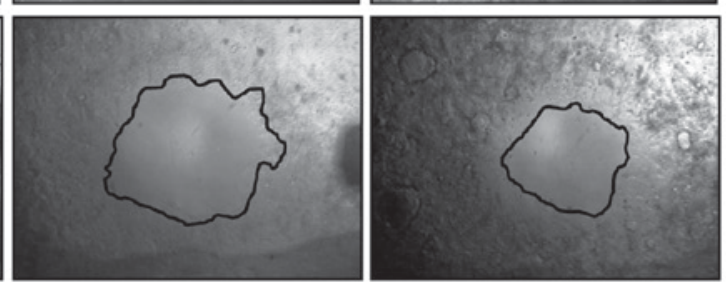

B

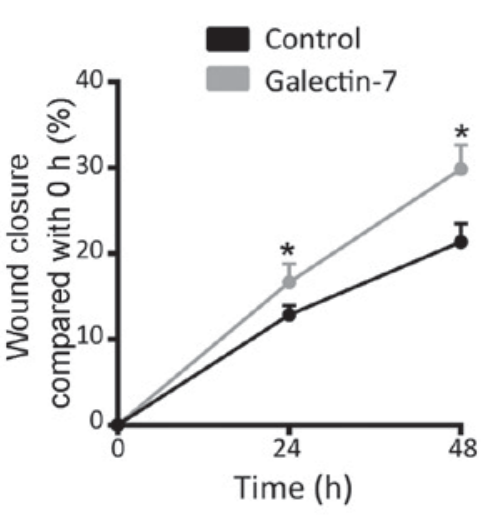

Figure 3. Galectin-7 enhances Ishikawa migration in an in vitro wound healing assay. (A) Ishikawa cells were grown to $100 \%$ confluence then wounded and in vitro repair was monitored after 24 and $48 \mathrm{~h}$. (B). Galectin-7 (1 $\mu \mathrm{g} / \mathrm{ml}$; grey) significantly enhanced Ishikawa cell migration, compared with vehicle control (black), after 24 and $48 \mathrm{~h}$. Significant difference was calculated by paired Student's t-test, ${ }^{*} \mathrm{P}<0.05 ; \mathrm{n}=4$; data are depicted as the mean \pm standard error of the mean.
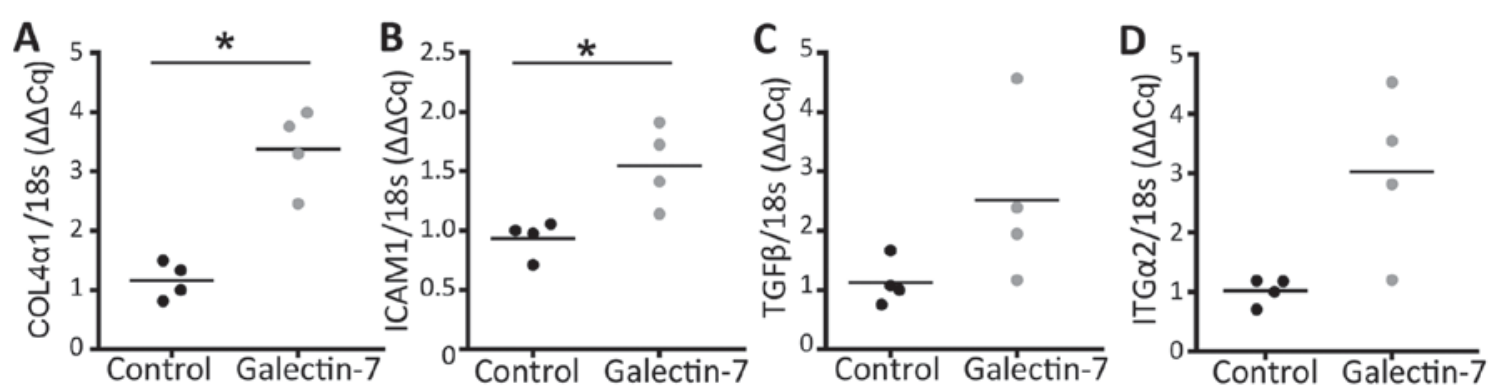

Figure 4. Galectin-7 enhances COL4 $\alpha 1$ and ICAM1 production by Ishikawa cells. Ishikawa cells were grown to $100 \%$ confluence and wounded prior to treatment with $1 \mu \mathrm{g} / \mathrm{ml}$ galectin-7 or vehicle control. Gene expression of (A) COL4 $\alpha 1$, (B) ICAM1, (C) TGF $\beta 1$ and (D) ITG $\alpha 2$ was determined after 24 h. Significant differences were calculated with a paired Student's t-test, ${ }^{*} \mathrm{P}<0.05 ; \mathrm{n}=4$. TGF $\beta 1$, transforming growth factor $\beta 1$; ITG $\alpha 2$, integrin subunit $\alpha 2$; ICAM1, intercellular adhesion molecule 1; COL $4 \alpha 1$, collagen type IV $\alpha 1$ chain.

cervical adenocarcinoma cells (27). Conversely, unlike the pro-apoptotic function of galectin-7 in colon and prostate cancer $(19,21)$ in the present study, it was determined that galectin-7 had no effect on apoptosis in EC cell lines. In breast cancer cells, galectin-7 is protective against apoptosis (16), a function that was not directly investigated in the present study. Why galectin-7 has differential roles in cancer is not well understood; however, the sub-cellular (nuclear, cytoplasmic or secreted) localization of galectin-7 may be important in determining its functional roles (8). The sub-cellular compartment that galectin-7 localizes to in EC remains to be determined. It is hypothesized that in vivo, elevated galectin-7 may promote invasiveness of $\mathrm{EC}$ within the uterus and also serve a role in the development of metastatic lesions.

Although galectin-7 production was significantly increased in Grade 3 EC (Fig. 1A and B), the levels were highly variable between women, indicating that Grade 3 EC should be separated into two cohorts: High; and moderate galectin-7 production. We previously demonstrated two cohorts of interleukin 11 production in Grade 3 EC (28). Galectin-7 production was also highly variable between the 3 EC cell lines tested (Fig. 1C), possibly reflecting the levels of galectin-7 in the original cancer from which these cell lines were generated. Given the high levels of galectin-7 produced by HEC1A cells, it is unsurprising that exogenous galectin-7 had no functional effect in these cells. It would be beneficial to investigate the effect of galectin-7 knockdown on HEC1A cells. The data indicates that EC with elevated galectin-7 production will exhibit decreased cell-cell adhesion and increased migration, potentially resulting in EC with greater tumorigenic potential. The association between EC metastasis and galectin-7 production should be investigated: Notably, the HEC1A cell line is also the most appropriate for generating metastatic EC in vivo (29).

Galectin-7 RNA and protein expression was elevated in $\mathrm{EC}$, compared with benign endometrium in the present study; however, the mechanism resulting in elevated galectin-7 in EC is not known. Galectin-7 is well known as a p53 inducible protein. In other cancer types, including breast and ovarian, galectin-7 expression is elevated due to a mutation in the p53 gene $(17,30)$. In breast cancer, this mutant p53 elevates galectin-7 via nuclear factor- $\kappa \mathrm{B}$ and tumor necrosis factor $\alpha$, without inducing apoptosis (30). In EC, p53 is mutated in $90 \%$ of type II serous carcinoma types, but only $11 \%$ of all type I endometrioid carcinoma types, although this rises to $20-30 \%$ for grade 3 cancer types (31). In the present study, elevated galectin-7 in type I endometrioid carcinoma (which is not characteristically associated with p53 mutations) was determined, indicating that p53 
mutation may not be the only mechanism to elevate galectin-7 in EC. The most common mutation in type I endometrioid cancer types is PTEN (31); however, there is no information regarding PTEN regulation of galectin-7 in any tissue. Altered methylation of galectin-7 is also associated with altered galectin-7 production in gastric cancer (20) and lymphoma (32). Although altered methylation is a feature of EC (31), there is no information regarding methylation of galectin-7 in EC. The role of estrogen should also be considered, due to Type I EC being characteristically estrogen-mediated; however, there is no information regarding whether estrogen regulates galectin-7 production. Further studies are required to determine the mechanism resulting in altered galectin-7 production in EC.

To mimic the rising levels of galectin-7 determined in EC, EC cell lines were treated with recombinant galectin-7 for the in vitro functional assays. The concentration used (1 $\mu \mathrm{g} / \mathrm{ml})$ was determined from the manufacturer's protocols and dose-response trials in previous studies $(14,15)$, but it is notable that extracellular galectin-7 remains to be identified in EC. Galectin-7 could not be detected in the endometrial epithelial EC cell line conditioned media (data not shown). Whether galectin-7 is secreted by EC tissue has not been investigated; however, exogenous galectin-7 is endocytozed into breast and ovarian cancer cells within minutes of application (33). Furthermore, extracellular galectin-7 upregulates intracellular galectin-7 production in breast and ovarian cancer cells (33), indicating that extracellular galectin-7 is able to exert its action via intra- and extra-cellular mechanisms. It was hypothesized that the recombinant galectin-7 utilized in the present study would have extra- and intra-cellular actions. We have previously demonstrated that endometrial epithelial cell lines display functional responses to exogenous galectin-7 (14,15). Whether exogenous galectin-7 is endocytozed into EC cells is yet to be determined: An increase in intracellular galectin-7 mRNA in Ishikawa cells $24 \mathrm{~h}$ after galectin-7 treatment was not identified (data not shown); however, only one time-point was used and intracellular protein was not measured.

Galectin-7 may promote tumorigenesis by altering the adhesive state of EC cells and promoting migration. To the best of our knowledge, the present study demonstrated for the first time that galectin-7 enhanced the gene expression of COL $4 \alpha 1$ and ICAM1, which are associated with cell adhesion and migration. COL $4 \alpha 1$ encodes the gene for the $\alpha$ chain of type IV collagen, a flexible basement membrane protein that assists in interactions with the basement membranes of nearby cells. COL $4 \alpha 1$ has not been previously associated with EC, except for a previous study indicating it is induced by Kruppel-like factor 9 in HEC1A cells (34). Supporting a role for COL $4 \alpha 1$ in EC invasion and metastasis, knockdown of COL $4 \alpha 1$ impairs invasion of urothelial carcinoma of the bladder (35). ICAM1 is a cell surface glycoprotein, which binds to integrins [cycle of differentiation 11 (CD11)/CD18] to promote cell-cell adhesion. ICAM1 is regulated by STAT1 in type II serous papillary EC (36) and is elevated in endometriosis (37). Our previous study using the endometrial epithelial ECC-1 cells to model endometrial luminal epithelium during menstrual repair demonstrated galectin-7 at $2.5 \mu \mathrm{g} / \mathrm{ml}$ enhanced ECC-1 wound closure via integrin binding and upregulation of $\beta$-catenin, contactin and TGF $\beta 1$. Notably, no significant effect of galectin-7 at $1 \mu \mathrm{g} / \mathrm{ml}$ on TGF $\beta 1$ expression was determined in Ishikawa cells in the present study, possibly due to the lower concentration of galectin-7 used. Despite this, the function of galectin-7 was identical between these two endometrial cell lines.

Although the xCELLigence data indicated that galectin-7 reduced cell attachment, experiments were not performed in the present study to confirm this; however, supporting an anti-adhesive role for galectin-7, we have previously demonstrated that exogenous galectin-7 impairs cell-cell adhesion between primary human endometrial epithelial cells (isolated from fertile females) and trophoblast cells (15). An effect of galectin-7 on EC adhesion and migration should be confirmed using primary EC cells.

In conclusion, elevated galectin-7 in EC may promote tumorigenesis via decreased cell attachment and increased migration. The role of galectin-7 in EC should be confirmed using primary EC cells and in vivo mouse models. Future studies should be directed towards determining the prognostic value of galectin-7 levels in serum or uterine fluid from females with EC. Serum galectin-7 concentration is a promising prospective biomarker for predicting subsequent miscarriage or the development of preeclampsia in pregnancy $(15,23)$. Galectin-7 may also have potential as a therapeutic target to treat EC, and inhibitors are currently being developed (38) and could be tested in mouse models of EC. Overall, the present study has identified galectin-7 as a novel mediator of EC progression.

\section{Acknowledgements}

The authors would like to thank the Victorian Cancer Biobank, in particular Ms Zdenka Prodanovic; and our research nurse Sister Judi Hocking (Hudson Institute of Medical Research) for the consenting of patients and collection of tissues.

\section{Funding}

This work was supported by the Victorian Government's Operational Infrastructure Support. Evdokia Dimitriadis was supported by NHMRC Senior Research Fellowship (grant no. 550905).

\section{Availability of data and materials}

Data sharing is not applicable to this article, as no datasets were generated or analyzed during the current study.

\section{Authors' contributions}

EM and ED conceived and designed the experiments. EM, MG, MVS, KR performed the experiments. EM, MVS and KN analyzed the data. EM wrote the manuscript, ED and MVS edited the manuscript.

\section{Ethics approval and consent to participate}

The present study was approved by the Monash Health Human Research and Ethics Committee (approval no. 06014C) and the Victorian Cancer Biobank (Project \#13018). Written and informed consent was obtained from each patient. 


\section{Patient consent for publication}

Patient consent for the publication of research generated from de-identified samples was obtained.

\section{Competing interests}

The authors declare that they have no competing interests.

\section{References}

1. Bray F, Ren JS, Masuyer E and Ferlay J: Global estimates of global cancer prevalence for 27 sites in the adult population in 2008. Int J Cancer 132: 1133-1145 2013.

2. Makker V, Green AK, Wenham RM, Mutch D, Davidson B and Miller DS: New therapies for advanced, recurrent and metastatic endometrial cancers. Gynecol Oncol Res Pract 4: 19, 2017.

3. Bilyk O, Coatham M, Jewer M and Postovit LM Epithelial-to-Mesenchymal transition in the female reproductive tract: From normal function to disease pathology. Front Oncol 7: 145,2017

4. Di Cristofano A and Ellenson LH: Endometrial Carcinoma. Annu Rev Pathol 2: 57-85, 2007.

5. Pecorelli S: Revised FIGO staging for carcinoma of the vulva, cervix, and endometrium. Int J Gynaecol Obstet 105: 103-104, 2009.

6. Elola MT, Wolfenstein-Todel C, Troncoso MF, Vasta GR and Rabinovich GA: Galectins: Matricellular glycan-binding proteins linking cell adhesion, migration and survival Cell Mol Life Sci 64: 1679-1700, 2007.

7. Dubé-Delarosbil C and St-Pierre Y: The emerging role of galectins in high-fatality cancers. Cell Mol Life Sci 75: 1215-1226, 2018.

8. Kaur M, Kaur T, Kamboj SS and Singh J: Roles of Galectin-7 in Cancer. Asian Pac J Cancer Prev 17: 455-461, 2016.

9. Lambropoulou M, Deftereou TE, Kynigopolos S, Patsias A, Anagnostopoulos C, Alexiadis G, Kotini A, Tsaroucha A Nikolaidou C, Kiziridou A, et al: Co-expression of Galectin-3 and CRIP-1 in endometrial cancer: Prognostic value and patient survial. Med Oncol 33: 8, 2016.

10. Saussez S and Kiss R: Galectin-7. Cell Mol Life Sci 63: 686-697, 2006.

11. Rabinovich GA and Toscano MA: Turning 'sweet' on immunity: Galectin-glycan interactions in immune tolerance and inflammation. Nat Rev Immunol 9: 338-352, 2009.

12. Cao Z, Said N, Amin S, Wu HK, Bruce A, Garate M, Hsu DK, Kuwabara I, Liu FT and Panjwani N: Galectins-3 and -7, but not Galectin-1, play a role in re-epithelialization of wounds. J Biol Chem 277: 42299-42305, 2002.

13. Cao Z, Said N, Wu HK, Kuwabara I, Liu FT and Panjwani N: Galectin-7 as a potential mediator of corneal epithelial cell migration. Arch Ophthalmol 121: 82-86, 2003.

14. Evans J, Yap J, Gamage T, Salamonsen L, Dimitriadis E and Menkhorst EM: Galectin-7 is important for nomal uterine repair following menstruation. Mol Hum Reprod 20: 787-798, 2014.

15. Menkhorst EM, Gamage T, Cuman C, Kaitu'u-Lino TJ, Tong S and Dimitriadis E: Galectin-7 acts as an adhesion molecule during implantation and increased expression is associated with miscarriage. Placenta 35: 195-201, 2014.

16. Demers M, Rose AA, Grosset AA, Biron-Pain K, Gaboury L, Siegel PM and St-Pierre Y: Overexpression of galectin-7, a myoepithelial cell marker, enhances spontaneous metastasis of breast cancer cells. Am J Pathol 176: 3023-3031, 2010.

17. Schulz H, Schmoeckel E, Kuhn C, Hofmann S, Mayr D, Mahner S and Jeschke U: Galectins-1, -3, and -7 are prognostic markers for survival of ovarian cancer patients. Int J Mol Sci 18: E1230, 2017.

18. Wang J, Liu Y, Yang Y, Xu Z, Zhang G, Liu Z, Fu H, Wang Z, Liu $\mathrm{H}$ and $\mathrm{Xu} \mathrm{J}$ : High expression of galectin-7 associates with poor overall survival of patients witn non-metastatic clear-cell renal cell carcinoma. Oncotarget 7: 41986-41995, 2016.

19. Labrie M, Vladoiu MC, Leclerc BG, Grosset AA, Gaboury L, Stagg J and St-Pierre Y: A mutation in the carbohydrate recognition domain drives a phenotypic switch in the role of Galectin-7 in prostate cancer. PLoS ONE 10: e0131307, 2015.
20. Kim SJ, Hwang JA, Ro JY, Lee YS and Chun KH: Galectin-7 is epigenetically-regulated tumor supressor in gastric cancer. Oncotarget 4: 1461-1471, 2013.

21. Ueda S, Kuwabara I and Liu FT: Suppression of tumor growth by galectin-7 gene transfer. Cancer Res 64: 5672-5676, 2004.

22. Livak K and Schmittgen TD: Analysis of relative gene expression data using real-time quantitative PCR and the 2(-Delta DeltaC(T)) method. Methods 25: 402-408, 2001.

23. Menkhorst EM, Koga K, Van Sinderen M and Dimitriadis E: Galectin-7 serum levels are altered prior to the onset of pre-eclampsia. Placenta 35: 281-285, 2014.

24. Winship AL, Van Sinderen M, Heffernan-Marks A and Dimitriadis E: Chondroitin sulfate proteoglycan protein is stimulated by interleukin 11 and promotes endometrial epithelial cancer cell proliferation and migration. Int J Oncol 50: 798-804, 2017.

25. Stefanowicz-Hajduk J, Adamska A, Bartoszewski R and Ochocka JR: Reuse of E-plate cell sensor arrays in the XCELLigence Real-Time Cell Analyzer. Biotechniques 61: 117-122, 2016.

26. Labrie M, Valdoiu MC, Grosset AA, Gaboury L and St-Pierre Y: Expression and functions of galectin-7 in ovarian cancer. Oncotarget 5: 7705-7721, 2014.

27. Park JE, Chang WY and Cho M: Induction of matrix metalloproteinase- 9 by galectin-7 through $\mathrm{p} 38 \mathrm{MAPK}$ signaling in HeLa human cervical epithelial adenocarcinoma cells. Oncol Rep 22: 1373-1379, 2009.

28. Yap J, Salamonsen LA, Jobling T, Nicholls PK and Dimitriadis E: Interleukin 11 is upregulated in uterine lavage and endometrial cancer cells in women with endometrial carcinoma. Reprod Biol Endocrinol 8: 63, 2010.

29. Winship AL, Van Sinderen M, Donoghue J, Rainczuk K and Dimitriadis E: Targeting interleukin-11 receptor- $\alpha$ impairs human endomterial cancer cell proliferation and invasion in vitro and reduces tumor growth and metastasis in vivo Mol Cancer Ther 15: 720-730, 2016.

30. Campion CG, Labrie M, Lavoie G and St-Pierre Y: Expression of galectin-7 is induced in breast cancer cells by mutant p53. PLoS One 8: e72468, 2013.

31. Piulats JM, Guerra E, Gil-Martin M, Roman-Canal B, Gatius S, Sanz-Pamplona R, Velasco A, Vidal A and Matias-Guiu X: Molecular approaches for classifying endometrial carcinoma. Gynecol Oncol 145: 200-207, 2017.

32. Demers M, Couillard J, Giglia-Mari G, Magnaldo T and St-Pierre Y: Increased galectin-7 gene expression in lymphoma cells is under the control of DNA methylation. Biochem Biophys Res Commun 387: 425-429, 2009.

33. Bibens-Laulan N and St-Pierre Y: Intracellular galectin-7 expression in cancer cell results from an autocrine transcriptional mechanism and endocytosis of extracellular galectin-7. PLoS ONE 12: e0187194, 2017.

34. Simmen FA, Su Y, Xiao R, Zeng Z and Simmen RC: The Kruppel-like factor 9 (KLF9) network in HEC-1-A endometrial carcinoma cells suggests the carcinogenic potential of dys-regulated KLF9 expression. Reprod Biol Endocrinol 6: 41, 2008.

35. Miyake M, Hori S, Morizawa Y, Tatsumi Y, Toritsuka M, Ohnishi S, Shimada K, Furuya H, Khadka VS, Deng Y, et al: Collagen type IV alpha 1 (COL4A1) and collagen type XIII alpha 1 (COL13A1) produced in cancer cells promote tumor budding at the invasion front in human urothelial carcinoma of the bladder. Oncotarget 8: 36099-36114, 2017.

36. Kharma B, Baba T, Matsumura N, Kang HS, Hamanishi J, Murakami R, McConechy MM, Leung S, Yamaguchi K, Hosoe Y, et al: STAT1 drives tumor progression in serous papillary endometrial cancer. Cancer Res 74: 6519-6530, 2014.

37. Kuessel L, Wenzl R, Proestling K, Balendran S, Pateisky P, Yotova 1st, Yerlikaya G, Streubel B and Husslein H: Soluble VCAM-1/soluble ICAM-1 ratio is a promising biomarker for diagnosing endometriosis. Hum Reprod 32: 770-779, 2017.

38. Vladoiu MC, Labrie M, Létourneau M, Egesborg P, Gagné D, Billard É, Grosset AA, Doucet N, Chatenet D and St-Pierre Y: Design of a peptidic inhibitor that targets the dimer interface of a prototypic galectin. Oncotarget 6: 40970-40980, 2015. 